\title{
Performance Analysis of Data Processing Architectures for the Smart Grid
}

\author{
Akshay Uttama Nambi S. N.*, Matteo Vasirani ${ }^{\dagger}$, R. Venkatesha Prasad*, Karl Aberer ${ }^{\dagger}$ \\ * Delft University of Technology (TUDelft), The Netherlands \\ $\dagger$ Ecole Polytechnique Fédérale de Lausanne (EPFL), Switzerland \\ \{akshay.narashiman,r.r.venkateshaprasad\}@tudelft.nl, \{matteo.vasirani, karl.aberer\}@epfl.ch
}

\begin{abstract}
Information and communication technology infrastructures play an important role to realize the full potential of Smart Grid (SG) applications. Several architectures proposed in the literature usually focus on communication requirement or data storage. However, it is still not clear which architecture best satisfies energy, storage, processing and communication requirements. The lack of understanding of key parameters, such as energy required, communication bandwidth, storage space, processing power, etc., has hindered the large scale SG deployments. In this paper, we investigate different data processing architectures for hierarchical power distribution networks. We introduce several key cost indicators to analyze hierarchical data processing architectures for the SG. In our evaluation, we consider realistic deployments in both dense and sparse environments and provide a detailed performance analysis of the proposed architectures. The results reported here are significant for SG designers, who can use them to discern the architecture that best fits the system requirements.
\end{abstract}

\section{INTRODUCTION}

Smart Grid (SG) is an intelligent power distribution system that uses information and communication technology (ICT) to enhance efficiency, reliability and sustainability of power generation and distribution network [1]. SG can be considered as an ensemble of several applications such as demand response, demand forecast, emergency management, anomaly detection and adaptive pricing, built upon an Advanced Metering Infrastructure (AMI) - a system that measures, collects and analyzes data about energy usage [2]. An estimate from a utility provider indicates 22 gigabytes of data being generated every day from its 2 million customers [3]. The overwhelming data generated by Smart Meters (SM) requires information management mechanisms for large scale data storage and processing. While there have been deployments of SG (e.g., Grid4EU $^{1}$ and SmartWatts ${ }^{2}$ ) with few participants, the design of suitable architecture to support envisaged SG applications on a large scale is an important research topic to be addressed [4].

In this paper we provide some comprehensive insights about certain key aspects, such as the energy usage to operate the architecture, the communication and storage requirements for large scale deployments, as well as the required processing capabilities. In particular, (i) we model different data processing architectures (centralized, decentralized, distributed and hybrid) for hierarchical power distribution networks; (ii) we

\footnotetext{
${ }^{1}$ http://www.grid4eu.eu/ ${ }^{2}$ http://www.smartwatts.de/
}

consider realistic SG deployments in both dense (i.e., urban) and sparse (i.e., rural) environments; (iii) we propose a generic approach to model and analyze several key cost indicators, such as energy consumption, processing power, storage requirements and communication bandwidth.

The remainder of the paper is organized as follows. Related literature is in Section II and different data processing architectures proposed are in Section III. Section IV describes various key cost indicators considered for evaluation. Section $\mathrm{V}$ presents our evaluation setup and simulation parameters considered. We describe the performance results of each architecture in Section VI and our conclusions are presented in Section VII.

\section{RELATED WORK}

In recent smart grid system deployments, smart meters collect data at an interval of 5 to 15 minutes, as compared to the traditional way that only records the meter data once a month [2]. Data values obtained as an average over a 15 minute interval may not be suitable to realize advanced applications such as distribution network automation and asset management, or disaggregation of appliance energy consumption data [5]. Thus smart meters in the near future will be required to measure values every $30 \mathrm{~s}$, posing a significant challenge for the operation of data processing architecture.

A secure decentralized data-centric information infrastructure for SG is proposed in [6] describing the challenges in low latency communication protocols, security and publishsubscribe data mechanisms for SG. A cloud-based SG information management model is proposed in [7] and [8], along with a discussion on key challenges. In contrast with the above works, we not only propose and analyze several architectures, but we also model important key cost indicators such as energy, communication, storage and processing based on the physical topology of the grid. Another cloud-based architecture for demand response (CDR) is proposed in [10]. CDR uses data-centric and publish/subscribe based communication for secure, scalable and reliable DR systems. Scalability aspects of data storage and monthly bill processing in SG is investigated in [4] and [9]. Several data storage mechanisms like centralized relational database, distributed relational database and file systems are compared and evaluated, as well as how communication cost scales with the number of smart meters and sampling frequency. 
To the best of our knowledge, there is no comprehensive analysis of different data processing architectures for SG that considers several key cost indicators like energy, communication, storage and processing. In this work, we provide a holistic approach to model and analyze all key cost indicators in urban and rural environments. The models developed in this work are generic and can be applied to any SG deployment.

\section{DATA PROCESSING ARCHITECTURES}

The current topology of the power distribution network is arranged according to the voltage. The distribution network is organized into multiple subgrids and consequently forming a hierarchical topology. In this paper, our architectural model adopts the hierarchical topology of the power distribution networks as described in [11]. The key elements of our architectural model are: (1) Home Area Nodes (HANs) - a node at the consumer premises that receives energy consumption information from all appliances in the household; (2) Neighborhood Area Nodes (NANs) - acts as an intermediate node between consumers and utility providers, and it serves a small geographical area (i.e., a neighborhood consisting of several houses). NAN receives information from the HANs within the neighborhood and multiple NANs are deployed to cover the utility's territory; (3) Utility Control Unit (UCU) - represents the central control entity of a utility company. This node is responsible for billing, maintaining data, determining electricity price and carrying out demand response management. UCU acts as the root node in our architectural model.

\section{A. Design Choices}

In our architectural model, HANs at each household periodically senses energy consumption and transmits it to the respective NAN, which acts as an intermediate node for the UCU. The interconnection between NANs and UCU is based on IEEE 802.16 (WiMAX) which supports a maximum data rate upto 1 Gbps [4]. Communication between NANs and HANs is based on $s u b-1 G H z$ transceivers which are best suited for both indoor and outdoor environments [12]. It should be noted that, the modeling and analysis in this paper can be further applied to any communication technology.

Data aggregation at the nodes can minimize the overall data communicated and also help in preserving sensitive information of customers. In this work, we consider timewise energy data aggregation (where consecutive time-stamped energy consumption readings are aggregated to reduce the granularity of the data collected) with different granularity. UCU can acquire information from the households by initiating a query. Nodes can respond to the query depending on their roles. By default, all nodes can send and receive a message, which is the minimum capability assumed at each node. The storage and processing icons in the Fig. 1, shows the additional capability available at each node depending on the architecture.

\section{B. Centralized Architecture}

In centralized architectures (Fig. 1a), only the UCU has data processing and storage capability. HANs periodically sense and transmit the energy consumption values to UCU via NANs making information flow uni-directional. No data aggregation is applied in centralized architectures.

\section{Decentralized Architecture}

In this architecture, only NANs have data processing and storage capabilities as shown in Fig. 1b. HANs transmit data periodically to the respective NAN, but instead of forwarding the data, the NAN stores and processes this data locally. In decentralized architectures, since complete data is available at the NANs, data aggregation is possible. For instance, NANs can aggregate hourly energy consumption while reporting to the UCU. UCU generates queries to retrieve information from NANs only when required. Thus, NANs act as central entities in this architecture.

\section{Distributed Architecture}

In distributed architectures, all HANs have data processing and storage capabilities. HANs periodically sense and store the energy consumption values locally. UCU initiates a query to fetch the data, which is forwarded to the NAN and in turn to the HANs. HANs process the query and send the reply to UCU via NAN. Thus, making the architecture distributed as illustrated in Fig. 1c. HANs are assumed to have sufficient data storage and processing capability and communicate only upon the reception of a query.

\section{E. Hybrid Architecture}

In hybrid architectures, HANs and NANs both have data processing and storage capabilities as shown in Fig. 1d. Hybrid architectures are extension of distributed architectures, where HANs not only sense and store but also transmits aggregated energy values to NANs. For instance, HANs can sense and store energy values periodically and at the end of the day send an aggregate energy consumption reading to the NAN. The data aggregation granularity may vary depending upon the application considered.

In the following section, we describe and model the key cost indicators used to evaluate the performance of architectures proposed.

\section{COST INDICATORS}

We propose a generic approach to model the key cost indicators such as energy consumption, processing power, storage and communication requirements to evaluate the performance of the proposed architectures.

\section{A. Energy consumption}

The energy required for the operation of the entire network (in Joules, J) includes the various activities the nodes can perform, such as reading from and writing into the storage, communicating, processing, etc. Energy consumption is calculated for the duration of one month. The energy consumed by HANs is,

$$
E_{\mathrm{HAN}}=E_{t}^{H \rightarrow N}+E_{r / w}^{H}+E_{p}^{H},
$$

where $E_{t}^{H \rightarrow N}$ is the energy consumed for communication from HAN to NAN, $E_{r / w}^{H}$ is the energy consumed for reading 


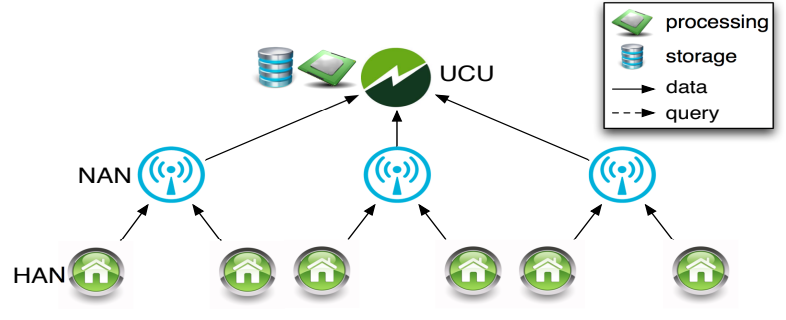

(a) Centralized Architecture

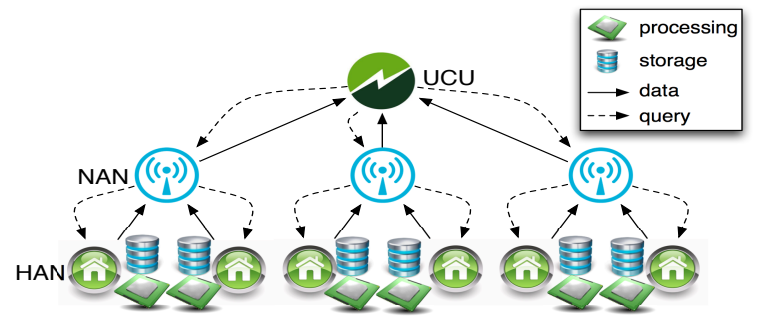

(c) Distributed Architecture

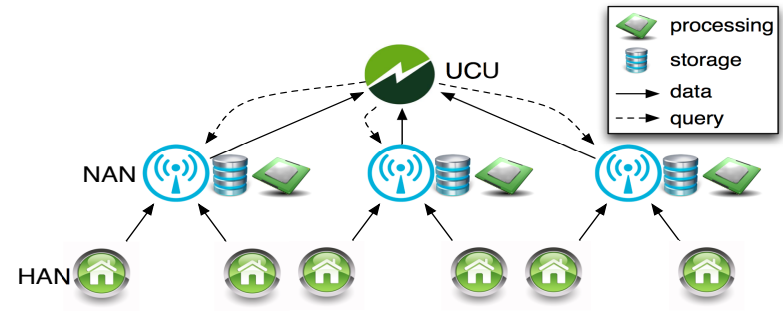

(b) Decentralized Architecture

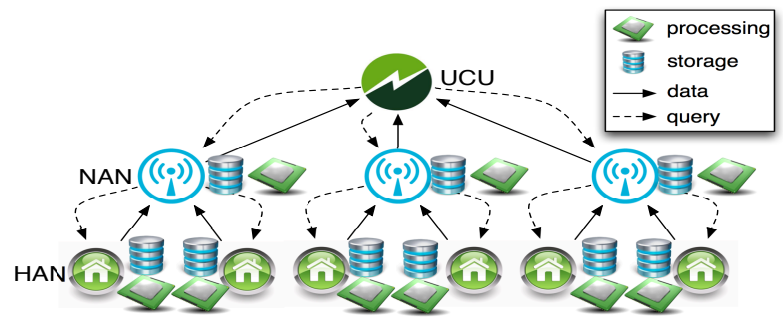

(d) Hybrid Architecture

Fig. 1: Data processing architectures for the smart grids.

from and writing into the storage, and $E_{p}^{H}$ is the energy consumed for processing.

The energy consumption for communication is,

$$
E_{t}^{H \rightarrow N}=e_{t x}^{H \rightarrow N} \ell_{t x}^{H \rightarrow N}+e_{r x}^{H \rightarrow N} \ell_{r x}^{H \rightarrow N},
$$

where $e_{t x}^{H \rightarrow N}$ (resp. $e_{r x}^{H \rightarrow N}$ ) is the energy required for transmission (resp. reception) of one byte of information between HAN and NAN, and $\ell_{t x}^{H \rightarrow N}$ (resp. $\ell_{r x}^{H \rightarrow N}$ ) is the length in bytes of the messages that have been transmitted (resp. received) by the HAN in one month. The energy consumption due to storage is defined as,

$$
E_{r / w}^{H}=e_{r}^{H} \ell_{r}^{H}+e_{w}^{H} \ell_{w}^{H},
$$

where $e_{r}^{H}$ (resp. $e_{w}^{H}$ ) is the energy required to read (resp. write) one byte of information, and $\ell_{r}^{H}\left(\operatorname{resp} . \ell_{w}^{H}\right)$ is the length in bytes of the messages that have been read from (resp. written into) the storage in one month. Finally, the energy consumption of processing is defined as,

$$
E_{p}^{H}=e_{p}^{H} n_{p}^{H},
$$

where $e_{p}^{H}$ represents the energy required for processing a byte at HAN, and $n_{p}^{H}$ is the number of processed bytes.

Similarly, energy consumption for a NAN is given by,

$$
E_{\mathrm{NAN}}=E_{t}^{H \rightarrow N}+E_{t}^{N \rightarrow U}+E_{r / w}^{N}+E_{p}^{N},
$$

where $E_{t}^{H \rightarrow N}$ is the energy consumed for communication between HANs and NANs, $E_{t}^{N \rightarrow U}$ is the energy consumed for communication between NANs and UCU, $E_{r / w}^{N}$ is the energy consumed for reading from and writing into the storage, and $E_{p}^{N}$ is the energy consumed for processing. These terms are defined as,

$$
\begin{gathered}
E_{t}^{H \rightarrow N}=e_{t x}^{H \rightarrow N} \ell_{t x}^{H \rightarrow N}+e_{r x}^{H \rightarrow N} \ell_{r x}^{H \rightarrow N} \\
E_{t}^{N \rightarrow U}=e_{t x}^{N \rightarrow U} \ell_{t x}^{N \rightarrow U}+e_{r x}^{N \rightarrow U} \ell_{r x}^{N \rightarrow U} \\
E_{r / w}^{N}=e_{r}^{N} \ell_{r}^{N}+e_{w}^{N} \ell_{w}^{N} ; \quad E_{p}^{N}=e_{p}^{N} n_{p}^{N} .
\end{gathered}
$$

Finally, for the UCU we have,

$$
E_{\mathrm{UCU}}=E_{t}^{N \rightarrow U}+E_{r / w}^{U}+E_{p}^{U} .
$$

The terms $E_{t}^{N \rightarrow U}$ (energy consumption for communication), $E_{r / w}^{U}$ (energy consumption for storage reading/writing) and $E_{p}^{U}$ (energy consumption for processing) are defined as,

$$
\begin{gathered}
E_{t}^{N \rightarrow U}=e_{t x}^{N \rightarrow U} \ell_{t x}^{N \rightarrow U}+e_{r x}^{N \rightarrow U} \ell_{r x}^{N \rightarrow U} \\
E_{r / w}^{U}=e_{r}^{U} \ell_{r}^{U}+e_{w}^{U} \ell_{w}^{U} ; \quad E_{p}^{U}=e_{p}^{U} n_{p}^{U} .
\end{gathered}
$$

Thus, the total energy consumption for the entire network in a month is,

$$
E_{\text {total }}=E_{\mathrm{UCU}}+\sum_{i \in \mathcal{N}} E_{\mathrm{NAN}}(i)+\sum_{j \in \mathcal{M}} E_{\mathrm{HAN}}(j),
$$

where $\mathcal{N}$ is the set of NANs and $\mathcal{M}$ is the set of HANs in the network. The total energy cost $C_{O}$ is the cost incurred for the operation of the entire network for one month period.

$$
C_{O}=E_{\text {total }} \cdot f_{E}
$$

where $E_{\text {total }}$ is the average energy (in Joules) required by all nodes to be operational for a period of one month, and $f_{E}$ is the price of energy (in $\$ /$ Joule).

\section{B. Processing}

The processing cost accounts for the number of operations (ops) required to respond to a query received by a node. The in-node operations required to respond to a query includes mainly arithmetic and relational operations. Processing cost depends on the number of messages to be processed and number of operations to be performed based on the query. The processing cost at node $k$, calculated for one month, is expressed as,

$$
P_{k}=\sum_{q \in \mathcal{Q}} n_{m} \cdot n_{q},
$$


where $\mathcal{Q}$ is the set of queries generated in the network, which depends on the supported applications, $n_{m}$ is the number of messages to be processed and $n_{q}$ represents the number of operations to be performed for query $q$. Given $P_{k}$, the resulting monetary cost for processing at node $k$ is,

$$
C_{P}(k)=P_{k} \cdot f_{P}\left(P_{k}\right),
$$

where $f_{P}(\cdot)$ is a non-linear function with respect to the number of operations to be performed that models the cost of processing (\$/ops) different from the cost of energy $f_{E}$ which is a constant. Thus, total processing cost of the network for one month is given as,

$$
C_{P}=C_{P}(\mathrm{UCU})+\sum_{i \in \mathcal{N}} C_{P}(i)+\sum_{j \in \mathcal{M}} C_{P}(j) .
$$

\section{Communication}

The communication cost accounts for the data rate (bits per second, bps) needed to transmit data from a HAN to the UCU through a NAN. Data rate for a HAN is expressed as,

$$
T_{\mathrm{HAN}}=\frac{8 \ell_{m}^{H \rightarrow N}}{t^{H \rightarrow N}}
$$

where $\ell_{m}^{H \rightarrow N}$ is the length of the message that has to be transmitted from the HAN to NAN, and $t^{H \rightarrow N}$ is the time period within which a HAN needs to transmit its information to the NAN. Given $T_{\mathrm{HAN}}$, the resulting monetary cost for communication at $\mathrm{HAN} j$ is,

$$
C_{T}(j)=T_{\mathrm{HAN}} \cdot f_{T}\left(T_{\mathrm{HAN}}\right)
$$

where $f_{T}(\cdot)$ is a non-linear function that models the cost of bandwidth (\$/bps). Similarly, data rate and resulting monetary cost for communication at NAN $i$ is expressed as,

$$
T_{\mathrm{NAN}}=\frac{8 \ell_{m}^{N \rightarrow U}}{t^{N \rightarrow U}}, C_{T}(i)=T_{\mathrm{NAN}} \cdot f_{T}\left(T_{\mathrm{NAN}}\right) .
$$

Therefore, the total communication cost required for transmission between HANs to NAN and NANs to UCU is expressed as,

$$
C_{T}=\sum_{i \in \mathcal{N}} C_{T}(i)+\sum_{j \in \mathcal{M}} C_{T}(j)
$$

\section{Storage}

The storage cost accounts for the total amount of storage capacity (in bytes) required by the node. The storage cost depends on the sampling interval $\tau$ and the time duration $\Delta T$ for which storage is needed. Thus, the storage requirement for a node $k$ is expressed as,

$$
S_{k}=\Delta T \frac{\ell_{m}}{\tau}
$$

where $\ell_{m}$ indicate the length of a message. Given $S_{k}$, the resulting monetary cost for storage at node $k$ is,

$$
C_{S}(k)=S_{k} \cdot f_{S}\left(S_{k}\right),
$$

where $f_{S}(\cdot)$ is a non-linear function that models the cost of storage (\$/byte). Thus, total storage cost of the network for one month is given by,

$$
C_{S}=C_{S}(\mathrm{UCU})+\sum_{i \in \mathcal{N}} C_{S}(i)+\sum_{j \in \mathcal{M}} C_{S}(j) .
$$

TABLE I: E=energy consumption, $\tau=$ sampling interval

\begin{tabular}{|c||c|c|c|c|}
\hline Queries generated (E/ $\tau$ ) & BAP & DR & DF & EM \\
\hline \hline E / 10 seconds & & $\mathrm{X}$ & $\mathrm{X}$ & \\
\hline E / 30 seconds & & $\mathrm{X}$ & & $\mathrm{X}$ \\
\hline $\mathrm{E} / 15$ minutes & & $\mathrm{X}$ & $\mathrm{X}$ & $\mathrm{X}$ \\
\hline $\mathrm{E} /$ hour & $\mathrm{X}$ & $\mathrm{X}$ & & \\
\hline E / day & $\mathrm{X}$ & $\mathrm{X}$ & $\mathrm{X}$ & $\mathrm{X}$ \\
\hline $\mathrm{E} /$ month & $\mathrm{X}$ & & $\mathrm{X}$ & $\mathrm{X}$ \\
\hline (Min, Max, Avg)E / day & & $\mathrm{X}$ & $\mathrm{X}$ & \\
\hline (Min, Max, Avg)E / month & $\mathrm{X}$ & & & $\mathrm{X}$ \\
\hline
\end{tabular}

TABLE II: Energy consumption for different operations. [14]

\begin{tabular}{cc}
\hline Operations & Energy consumption \\
\hline \hline Transmission @ sub-1GHz & $0.164 \mathrm{~mJ} / \mathrm{byte}$ \\
Reception @ sub-1GHz & $0.08 \mathrm{~mJ} / \mathrm{byte}$ \\
Transmission @IEEE 802.16 & $0.324 \mathrm{~mJ} /$ byte \\
Reception @IEEE 802.16 & $0.100 \mathrm{~mJ} /$ byte \\
Read from flash & $0.09 \mu \mathrm{J} / \mathrm{byte}$ \\
Write to flash & $0.8 \mu \mathrm{J} / \mathrm{byte}$ \\
Processing & $0.14 \mu \mathrm{J} / \mathrm{byte}$ \\
\hline
\end{tabular}

\section{Evaluation Setup}

Billing and adaptive pricing (BAP), Demand response (DR), Demand forecast (DF) and Emergency Management (EM) applications are considered in our analysis. These applications have different data requirements, which imply different data acquisition queries generated by the UCU. The queries and the requirements for each application are described in Table I.

\section{A. Environment}

In our cost-benefit analysis we consider two environments: urban and rural. Average total population in an urban environment is around $4.8 \mathrm{M}$ with $1.6 \mathrm{M}$ households and to provide adequate coverage, 73 NANs are required as described in [13]. Rural environments have total population of $1.4 \mathrm{M}$ with $476 \mathrm{~K}$ households and 76 NANs operating at sub- $1 \mathrm{GHz}$ are required to provide full coverage [13].

\section{B. Simulation parameters}

In this work, a standard wireless sensor node (WSN) is considered as HAN and its configuration depends on the architecture. Each HAN samples data by default every 5 minutes, which can be programmed based on the requirement or upon reception of the query. Each HAN is associated with a sub- $1 \mathrm{GHz}$ transceiver to communicate with the NAN. Similarly, NANs are equipped with both sub-1GHz and WiMAX transceivers to communicate with HANs and UCU. Table II shows the energy consumption for different operations performed by the HAN. Message size of data, query, query-reply, aggregated messages are considered to be 50, 5, 10 and 10 bytes respectively. 
TABLE III: Energy consumption (urban, 1.6M HANs).

\begin{tabular}{ccccc}
\hline Architectures & Storage & Proc. & Comm. & Total Energy \\
\hline \hline Centralized & $4 \%$ & $12.6 \%$ & $83.4 \%$ & $554.0 \mathrm{MJ}$ \\
Decentralized & $2.3 \%$ & $16.2 \%$ & $81.5 \%$ & $213.5 \mathrm{MJ}$ \\
Distributed & $15.9 \%$ & $20.4 \%$ & $63.7 \%$ & $19.8 \mathrm{MJ}$ \\
Hybrid & $7.1 \%$ & $3 \%$ & $89.9 \%$ & $9.6 \mathrm{MJ}$ \\
\hline
\end{tabular}

\section{Vi. Performance Results}

\section{A. Energy consumption}

Energy consumption cost per architecture for a urban environment $^{3}$ is described in Table III. It is evident that centralized architecture consumes significant amount of energy as all data needs to be relayed to the UCU. Distributed and hybrid architectures consume much less energy compared to centralized and decentralized architectures. The significant energy savings in distributed approaches is due to the reduced number of transmissions. Energy consumption of the hybrid architecture is the lowest compared to all other architectures. This energy saving is achieved by sending aggregated data to NANs as compared to storing data only at HANs, as in distributed architecture.

In general, the total energy consumption increases rapidly as the number of houses increases for centralized and decentralized architectures. In case of distributed and hybrid architectures, the increase in energy consumption is very gradual, thus increasing their scalability. Similar trends are obtained for rural environments. We remark that the energy consumption considers only communication, processing and storage operations, although other factors could be considered, such as cooling, lights, etc. It is evident that the most significant energy factor in all the architectures is communication. Hence, reducing communication can in turn reduce overall energy consumption, as can be seen in distributed and hybrid architectures.

\section{B. Processing}

Processing accounts for the number of operations performed to respond to a query as described in Section IV-B. Processing requirements depend on the number of messages the node has to process before replying. In this work, only arithmetic and relational operations are considered for processing cost at HANs and NANs. The results for one month duration in an urban environment with 1.6M HANs is shown in Table IV. Processing is performed only at UCU in a centralized architecture, only at NANs in a decentralized architecture, only at HANs in a distributed architecture and at both HANs and NANs in a hybrid architecture.

In centralized architectures, since UCU performs all processing, the processing requirements increase with the number of houses. In a decentralized architecture, processing is performed at each NAN and it's three order of magnitude lower

\footnotetext{
${ }^{3}$ In our experimental evaluation we considered two cases: (i) each NAN has the same number of HANs \& (ii) each NAN has a uniformly distributed random number of HANs. We found that there is not much difference in energy consumption between the two cases. Thus, for simplicity we consider equal number of HANs being allocated to each NAN.
}

TABLE IV: Processing operations (urban, 1.6M HANs).

\begin{tabular}{lc}
\hline Architectures & Number of operations \\
\hline \hline Centralized & $5400 \mathrm{M}$-ops \\
Decentralized & $1800 \mathrm{M}$-ops \\
Distributed & $0.36 \mathrm{M}$-ops \\
Hybrid - NAN & $31.5 \mathrm{M}$-ops \\
Hybrid - HAN & $0.008 \mathrm{M}$-ops \\
\hline
\end{tabular}

TABLE V: Bandwidth required for various architectures.

\begin{tabular}{lllll}
\hline \multirow{2}{*}{ Architectures } & \multicolumn{2}{c}{ Urban } & \multicolumn{2}{c}{ Rural } \\
& HAN & NAN & HAN & NAN \\
\hline \hline Centralized & $480 \mathrm{bps}$ & $11 \mathrm{Mbps}$ & $480 \mathrm{bps}$ & $3 \mathrm{Mbps}$ \\
Decentralized & $480 \mathrm{bps}$ & $32 \mathrm{Mbps}$ & $480 \mathrm{bps}$ & $1 \mathrm{Mbps}$ \\
Distributed & $144 \mathrm{bps}$ & $3 \mathrm{Mbps}$ & $144 \mathrm{bps}$ & $1 \mathrm{Mbps}$ \\
Hybrid & $138 \mathrm{bps}$ & $2 \mathrm{Mbps}$ & $138 \mathrm{bps}$ & $0.5 \mathrm{Mbps}$ \\
\hline
\end{tabular}

than in a centralized architecture. In distributed architectures, the number of operations at each HAN is reduced by order of four compared to decentralized architecture. In hybrid architectures, processing effort is distributed at both HANs and NANs and has the least processing cost at each HAN. Similar trends are also observed for rural environments.

\section{Communication}

The communication cost as described in Section IV-C is the average data rate required to support the SG applications. The time of reference $t^{H \rightarrow N}$ and $t^{N \rightarrow U}$ in Eq. (14) and Eq. (16) are considered to be $1 \mathrm{~s}$. Table $\mathrm{V}$ shows the average bandwidth requirement at each HAN and NAN for both urban and rural environments with $1.6 M$ HANs and $476 K$ HANs respectively. Data rate requirements for a HAN is same, irrespective of the environment, as each HAN transmits the same data based on the architecture selected. However, the data rate required at NANs in urban environment is higher than rural environment, since more HANs are associated with each NAN in an urban environment. The needed bandwidth between HANs and NAN is higher for centralized and decentralized architectures, since HANs has no storage and all data messages are forwarded to NAN. However, since distributed storage and processing is adopted in distributed and hybrid architectures, the number of transmissions performed at the HAN is reduced. Thus, the bandwidth requirement is significantly reduced in these architectures. Similar trends with scaled-down bandwidth requirements are observed for rural environments.

\section{Storage}

Storage required by each node for different architectures in urban environment, for a duration $\Delta T=1$ year is shown in Fig. 2a. The default sampling interval of 5 minutes is considered to determine the storage cost as described in Eq. (18). As with other costs, storage cost for centralized architecture is the highest compared to other architectures, as all data is stored at the UCU. The storage required by other architectures is much less than in a centralized architecture, with distributed architecture having the lowest. As the number of HANs increase, the required storage increases linearly 


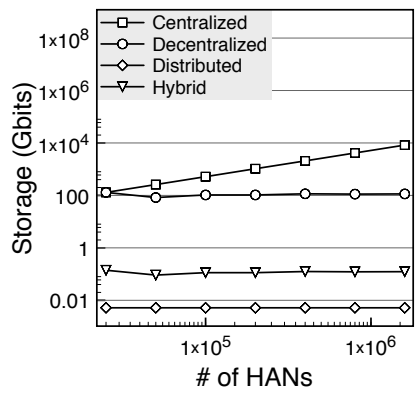

(a) \# of HANs

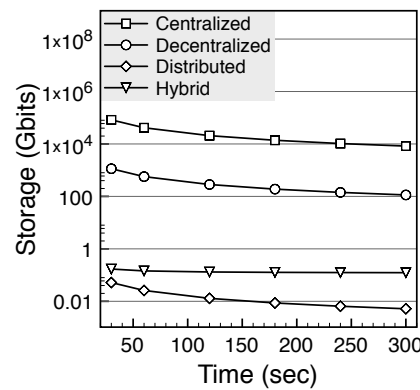

(b) Sampling Interval
Fig. 2: Required storage in urban environment.

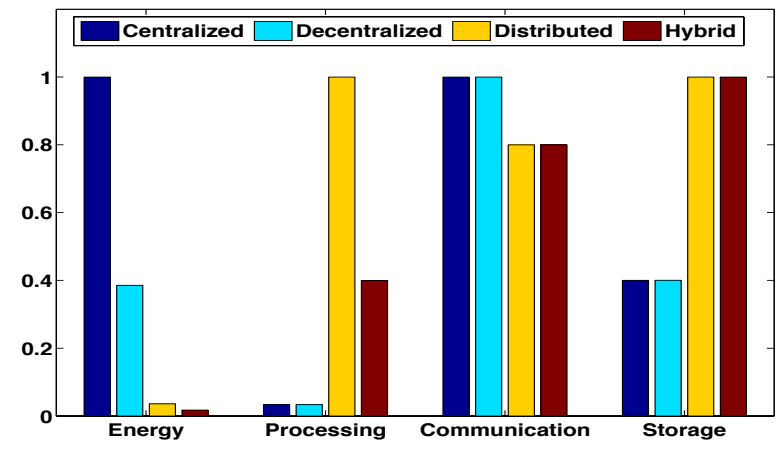

Fig. 3: Cost distribution among various architectures.

in centralized architectures. On the other hand, since equal number of HANs are allocated to each NAN, for all the other architectures storage is constant, as seen in the Fig. 2a. Thus distributed storage is more scalable with increase in number of HANs compared to single point centralized storage. Finally, the required storage as a function of sampling interval is shown in Fig. 2b. Higher sampling intervals indicate less frequent sensing of energy values. Storage cost decreases with increase in sampling interval, regardless of the architecture.

\section{E. Cost Distribution}

Figure 3 shows the normalized cost distribution for the key cost indicators across various architectures. Energy, processing, communication and storage costs are calculated as described in Eq. (10), (13), (17) and (20) respectively. Cost indicators are normalized across each cost factor. Centralized architectures has low cost for processing, communication and storage elements (which accounts for one time deployment cost) but with high operational energy cost. Since, all data is stored and processed at one location it suffers from single point of failure and is less scalable. However, distributed architectures have low operational energy cost but with high deployment cost due to distributed processing and storage. Hybrid architectures have the least energy requirement but with high deployment cost due to storage and processing elements at both HANs and NANs. However, decentralized architectures has higher energy cost compared to hybrid but with low deployment cost since only NANs has storage and processing elements.

Thus, the choice of architecture could be to have a more energy efficient architecture or highly scalable distributed architecture with high deployment cost or simple less scalable centralized architecture and depends upon the objective of the implementation.

\section{CONCLUSIONS}

This paper introduced several key cost indicators to analyze different ICT architectures for communication, storage and processing of Smart Grid data. The proposed architectures have been evaluated in urban and rural environments, based on energy consumption, processing power, storage requirements and communication bandwidth. The results showed that centralized architectures are the cheapest to deploy. Distributed architectures on the other hand have a higher deployment cost but are more energy efficient. In this paper we proposed two alternative architectures that make use of data aggregation to reduce deployment cost. This quantitative evaluation will help Smart Grid designers to select the architecture that best fits their system requirements and deployment scenarios.

\section{REFERENCES}

[1] X. Fang, S. Misra, G. Xue, and D. Yang. Smart grid - the new and improved power grid: A survey. IEEE Communications Surveys and Tutorials, 14(4):944-980, 2012.

[2] U.S. Dept. of Energy. Advanced metering infrastructure. February 2008, [Online] https://www.smartgrid.gov/sites/default/files/pdfs/advanced_ metering _infrastructure_02-2008.pdf.

[3] $\bar{M}$. Shargal and D. Houseman. The big picture of your coming smart grid, 2009, [Online] http://www.smartgridnews.com/artman/publish/commentary/The_Big Picture_of_Your_Coming_Smart_Grid-529.html.

[4] J. Zhou, R. Hu, and Y. Qian. Scalable distributed communication architectures to support advanced metering infrastructure in smart grid. Parallel and Distributed Systems, IEEE Transactions on, 2012.

[5] S. Akshay Uttama Nambi, T. G. Papaioannou, D. Chakraborty, and K. Aberer. Sustainable energy consumption monitoring in residential settings. In INFOCOM, Proceedings, IEEE, 2013.

[6] Y.-J. Kim, M. Thottan, V. Kolesnikov, and W. Lee. A secure decentralized data-centric information infrastructure for smart grid. Communications Magazine, IEEE, 2010.

[7] S. Rusitschka, K. Eger, and C. Gerdes. Smart grid data cloud: A model for utilizing cloud computing in the smart grid domain. In IEEE SmartGridComm, 2010

[8] X. Fang, D. Yang, and G. Xue. Evolving smart grid information management cloudward: A cloud optimization perspective. Smart Grid, IEEE Transactions on, 4(1):111-119, 2013.

[9] M. Arenas-Martinez, S. Herrero-Lopez, A. Sanchez, J. Williams, P. Roth, P. Hofmann, and A. Zeier. A comparative study of data storage and processing architectures for the smart grid. In IEEE SmartGridComm, 2010.

[10] H. Kim, Y.-J. Kim, K. Yang, and M. Thottan. Cloud-based demand response for smart grid: Architecture and distributed algorithms. In IEEE SmartGridComm, 2011.

[11] Rong Yu; Yan Zhang; Gjessing, S.; Chau Yuen; Shengli Xie; Guizani, M., Cognitive radio based hierarchical communications infrastructure for smart grid, Network, IEEE , vol.25, no.5, pp.6,14, September, 2011.

[12] S. Aust, R. Prasad, and I. Niemegeers. Performance evaluation of sub $1 \mathrm{ghz}$ wireless sensor networks for the smart grid. In IEEE Local Computer Networks (LCN), 2012.

[13] Electric Power Research Institute, Wireless Field Area Network Spectrum Assessment, 2010.[Online]

[14] T. Prabhakar, S. N. Akshay, R. Venkatesha Prasad, S. Shilpa, K. Prakruthi, and I. Niemegeers. A distributed smart application for solar powered wsns. In NETWORKING, LNCS, Springer, 2012. 